\title{
WHICH QUARTIC POLYNOMIALS HAVE A HYPERBOLIC ANTIDERIVATIVE?
}

\author{
RAJESH PEREIRA
}

In Memory of Serguei Shimorin

\begin{abstract}
Every linear, quadratic or cubic polynomial having all real zeros is the derivative of a polynomial having all real zeros. The statement is false for higher degree polynomials. In particular, not every fourth degree polynomial with real zeros is the derivative of a polynomial having all real zeros. We derive a necessary and sufficient condition for a quartic polynomial to be the derivative of a polynomial having all real zeros. This condition is a single quadratic form inequality involving the zeros of the quartic polynomial.
\end{abstract}

\section{INTRODUCTION}

The relationship between the zeros of a polynomial and those of its derivative has been of significant interest to mathematicians for at least three centuries. Serguei Shimorin has worked in this area 4. In this paper, we will study polynomials having all of their zeros on the real line; these are sometimes called hyperbolic polynomials. It is a simple consequence of Rolle's theorem that the derivative of a hyperbolic polynomial is a hyperbolic polynomial.

The converse to this is false. A hyperbolic polynomial of degree three or less always has a hyperbolic antiderivative. However for $n \geq 4$, there are $n$th degree hyperbolic polynomials which have no hyperbolic antiderivatives at all. The example $p(x)=(x-1)^{2}(x-4)^{2}$ was given in [1].

It would be desirable to have a systematic test for this. Suppose $Q(z)$ is an $(n+1)$ th degree monic hyperbolic polynomial with zeros $z_{1} \geq z_{2} \geq z_{3} \geq \ldots \geq z_{n} \geq$ $z_{n+1}$. Then $Q(z)$ is nonnegative on $\left[z_{2 j+1}, z_{2 j}\right]$ and nonpositive on $\left[z_{2 j}, z_{2 j-1}\right]$ for all $j: 1 \leq j \leq \frac{n}{2}$. Now let $w_{1} \geq w_{2} \geq w_{3} \geq \ldots \geq w_{n}$ be the zeros of $Q^{\prime}(z)$. By Rolle's theorem $z_{j+1} \leq w_{j} \leq z_{j}$ and hence $Q\left(w_{2 j}\right) \geq 0$ and $Q\left(w_{2 j-1}\right) \leq 0$ for all $j$. Conversely if $Q$ is a real monic $(n+1)$ th degree polynomial and there exists $w_{1} \geq w_{2} \geq w_{3} \geq \ldots \geq w_{n}$ with $Q^{\prime}\left(w_{j}\right)=0, Q\left(w_{2 j}\right) \geq 0$ and $Q\left(w_{2 j-1}\right) \leq 0$ for all $j$, then $Q$ is hyperbolic by the Intermediate Value theorem.

This is a nice characterization, however it is in terms of $Q(z)$. If we start with the polynomial $p(z)$ and find an antiderivative, there is no guarantee that we will get the particular $Q(x)$ which has all of its zeros real, we will instead get $P(z)=Q(z)+c$

Date: June 29, 2018.

1991 Mathematics Subject Classification. Primary 26C10; Secondary 26D05.

Key words and phrases. geometry of polynomials, hyperbolic polynomials, quartics.

This work supported by NSERC and the hospitality of the Mittag-Leffler Institute. I would like to thank Profs. Vladimir Kostov and Alan Horwitz for their suggestions which led to this second version of this ArXiv paper. 
for some arbitrary real $c$. This will shift everything by $c$ which gives us the criterion of Souroujon and Stoyanov.

Lemma 1.1. 7] Let $\left\{w_{k}\right\}_{k=1}^{n}$ be real numbers with $w_{1} \geq w_{2} \geq \ldots \geq w_{n-1} \geq w_{n}$. Let $p(x)=\prod_{k=1}^{n}\left(x-w_{k}\right)$ and let $P(x)$ be any antiderivative of $p(x)$, then there exists $c \in \mathbb{R}$ such that $P(x)-c$ has all zeros real if and only if $\max \left\{P\left(w_{k}\right): k\right.$ odd $\} \leq \min \left\{P\left(w_{k}\right): k\right.$ even $\}$ in which case we can take any choice of $c$ such that $\max \left\{P\left(w_{k}\right): k\right.$ odd $\} \leq c \leq \min \left\{P\left(w_{k}\right): k\right.$ even $\}$.

We can restate this Lemma in a more convenient form.

Lemma 1.2. Let $\left\{w_{k}\right\}_{k=1}^{n}$ be real numbers with $w_{1} \geq w_{2} \geq \ldots \geq w_{n-1} \geq w_{n}$. Let $p(x)=\prod_{k=1}^{n}\left(x-w_{k}\right)$ and let $P(x)$ be any antiderivative of $p(x)$, then there exists $c \in \mathbb{R}$ such that $P(x)-c$ has all zeros real if and only if $P\left(w_{j}\right) \geq P\left(w_{k}\right)$ whenever $j$ is even and $k$ is odd and $|j-k| \geq 3$.

We note that if $j-k=1$, then $p(x)>0$ on the interval $\left(w_{k}, w_{j}\right)$ and if $k-j=1$, then $p(x)<0$ on the interval $\left(w_{j}, w_{k}\right)$; therefore in both cases we automatically get $P\left(w_{j}\right) \geq P\left(w_{k}\right)$ which is why we can drop these as conditions in Lemma 1.2 (Interestingly, while this fact will not play a role in this paper, these inequalities are the only conditions on the ordered set $\left\{P\left(w_{j}\right)\right\}$ for arbitrary hyperbolic polynomials $P$. See 2] for the exact statement, proof and discussion of this fact.)

\section{Quartic Polynomials}

A simple induction shows that the number of inequalities in Lemma 1.2 is $\left\lfloor\left(\frac{n}{2}-1\right)^{2}\right\rfloor$ when $n \geq 2$. In particular, we see that for fourth degree polynomials the existence of a hyperbolic antiderivative essentially is equivalent to a single condition. We state this special case of Lemma 1.2

Corollary 2.1. Let $\left\{w_{k}\right\}_{k=1}^{n}$ be real numbers with $w_{1} \geq w_{2} \geq w_{3} \geq w_{4}$. Let $p(x)=\prod_{k=1}^{4}\left(x-w_{k}\right)$ and let $P(x)$ be any antiderivative of $p(x)$, then there exists $c \in \mathbb{R}$ such that $P(x)-c$ has all zeros real if and only if $P\left(w_{4}\right) \geq P\left(w_{1}\right)$.

We note that if $a$ and $b$ are real numbers with $a \neq 0$ then $p(a x+b)$ is a hyperbolic polynomial with a hyperbolic antiderivative if and only if $p(x)$ is. We may therefore apply the transformation $a x+b$ which maps $w_{1}$ to 1 and $w_{4}$ to -1 and consider quartic polynomials having $1,-1, s$ and $t$ as zeros with $s, t \in[-1,1]$. In this case, we get a very simple condition in terms of the zeros of $p$.

Theorem 2.2. Let $s, t \in[-1,1]$ and let $p(x)=(x-1)(x-s)(x-t)(x+1)$. Then $p(x)$ has a hyperbolic antiderivative if and only if st $\geq-\frac{1}{5}$.

Proof. Since $p(x)=x^{4}-(s+t) x^{3}+(s t-1) x^{2}+(s+t) x-s t$, we get $60 P(x)=$ $12 x^{5}-15(s+t) x^{4}+20(s t-1) x^{3}+30(s+t) x^{2}-60 s t x$ where $P(x)$ is an antiderivative of $p(x)$. Now $60(P(1)-P(-1))=2(12+20(s t-1)-60 s t)=8(3+5(s t-1)-15 s t)=$ $8(-2-10 s t)=-16(1+5 s t)$; which means $p$ has a hyperbolic antiderivative if and only if $s t \geq-\frac{1}{5}$.

We note that the mapping $\frac{w_{1}-w_{4}}{2} x-\frac{w_{1}+w_{4}}{2}$ maps the numbers $-1, s, t, 1$ (where $s=\frac{2 w_{2}-w_{1}-w_{4}}{w_{1}-w_{4}}$ and $\left.t=\frac{2 w_{3}-w_{1}-w_{4}}{w_{1}-w_{4}}\right)$ to $w_{1}, w_{2}, w_{3}, w_{4}$. The inequality $s t \geq-\frac{1}{5}$ is equivalent to $5\left(2 w_{2}-w_{1}-w_{4}\right)\left(2 w_{3}-w_{1}-w_{4}\right)+\left(w_{1}-w_{4}\right)^{2} \geq 0$. After some algebra, we can restate this condition as follows: 
Theorem 2.3. Let $\left\{w_{i}\right\}_{i=1}^{4}$ be real numbers with $w_{1} \geq w_{2} \geq w_{3} \geq w_{4}$ and let $p(x)=\left(x-w_{1}\right)\left(x-w_{2}\right)\left(x-w_{3}\right)\left(x-w_{4}\right)$. Then $p(x)$ has a hyperbolic antiderivative if and only if $w^{t} A w \geq 0$ where $w=\left(w_{1}, w_{2}, w_{3}, w_{4}\right)$ and where

$$
A=\left[\begin{array}{cccc}
6 & -5 & -5 & 4 \\
-5 & 0 & 10 & -5 \\
-5 & 10 & 0 & -5 \\
4 & -5 & -5 & 6
\end{array}\right]
$$

We can also reformulate this result in terms of the gaps between the zeros. Let $g_{j}=w_{j}-w_{j+1}$ for $j=1,2,3$. Then $5\left(2 w_{2}-w_{1}-w_{4}\right)\left(2 w_{3}-w_{1}-w_{4}\right)+\left(w_{1}-w_{4}\right)^{2}=$ $5\left(-g_{1}+g_{2}+g_{3}\right)\left(-g_{1}-g_{2}+g_{3}\right)+\left(g_{1}+g_{2}+g_{3}\right)^{2}=5\left(g_{3}-g_{1}\right)^{2}-5 g_{2}^{2}+\left(g_{1}+g_{2}+g_{3}\right)^{2}$. This gives us the following result.

Theorem 2.4. Let $\left\{w_{i}\right\}_{i=1}^{4}$ be real numbers with $w_{1} \geq w_{2} \geq w_{3} \geq w_{4}$ and let $p(x)=\left(x-w_{1}\right)\left(x-w_{2}\right)\left(x-w_{3}\right)\left(x-w_{4}\right)$. Then $p(x)$ has a hyperbolic antiderivative if and only if $v^{t} B v \geq 0$ where $v=\left(w_{1}-w_{2}, w_{2}-w_{3}, w_{3}-w_{4}\right)$ is the vector of distances between adjacent zeros of $p$ and where

$$
B=\left[\begin{array}{ccc}
6 & 1 & -4 \\
1 & -4 & 1 \\
-4 & 1 & 6
\end{array}\right]
$$

This suggests the problem of finding the characterization of the zero sets of higher degree polynomials which have hyperbolic antiderivatives. It is clear that the characterization will be a set of homogeneous polynomial inequalities of the form $S_{n}=\left\{w_{1} \geq w_{2} \geq \ldots \geq w_{n}: p_{n, k}\left(w_{1}, w_{2}, \ldots, w_{n}\right) \geq 0 ; 1 \leq k \leq\left\lfloor\left(\frac{n}{2}-1\right)^{2}\right\rfloor\right\}$. The degrees of these polynomials are less than or equal to $n-2$.

It is interesting to note that a related problem has been fairly well studied. A polynomial $p$ is said to be very hyperbolic if it is the $n^{\text {th }}$ derivative of a hyperbolic polynomial for all natural numbers $n$. V. P. Kostov has extensively studied the geometry of the sets of very hyperbolic polynomials; see the references [3, 5, 6] for more details.

\section{REFERENCES}

[1] G.G. Bilodeau. Generating polynomials all of whose roots are real. Mathematics Magazine, 64(4):263-270, 1991.

[2] C. Davis. Problem 4714. Amer. Math. Monthly, 63(10):729-729, 1956.

[3] Dimitar K Dimitrov and Vladimir P Kostov. Sharp Turán inequalities via very hyperbolic polynomials. Journal of Mathematical Analysis and Applications, 376(2):385-392, 2011.

[4] D. Khavinson, R. Pereira, M. Putinar, E. B. Saff, and S. Shimorin. Borcea's variance conjectures on the critical points of polynomials. In Notions of Positivity and the Geometry of Polynomials, pages 283-309. Springer, 2011.

[5] Vladimir Kostov. Topics on hyperbolic polynomials in one variable. SMF, 2011.

[6] Vladimir Petrovich Kostov. Very hyperbolic polynomials. Functional Analysis and Its Applications, 39(3):229-232, 2005.

[7] D. M. Souroujon and T. S. Stoyanov. About the primitive polynomials of polynomials with real zeros. Journal of Analysis and Applications, 14(1):21-31, 2016.

Department of Mathematics and Statistics, University of Guelph, Guelph ON. N1G 2W1, CANADA

E-mail address: pereirar@uoguelph.ca 